\title{
Simplified Calculus to Estimate the Acoustical Absorption of Non- Planar Materials
}

\author{
J. Pfretzschner, F. Simón, R. Mª Rodríguez, C. de la Colina \\ Instituto de Acústica (CSIC). C. Serrano, 14428006 Madrid, Spain
}

Summary: A simplified model for the acoustical absorption coefficient valid for non-planar surfaces of porous material is developed. This model is tested with experimental arrangements for granular specimens

\section{INTRODUCTION}

The use of absorbent noise barriers is a real need in cases where the traffic line is surrounded by inhabited areas. In this work we are interested in granular absorbent materials. The absorption curve, as a function of the frequency, for hard-backed layers of granular materials, shows undesirable series of maxima and minima related to the layer deep. In order to increase the efficiency of these absorbing devices, it is necessary to smooth their frequency response curve. It is obvious that one of the easiest solutions consists of modifying the layer surface with selected profiles (e.g., wedges). Absorption of wedges was studied in 1946 by Beranek (1), but their results are valid for fiber glass only and what is more, they are obsolete due to the change on the properties of modern fiber glass. An option is the use of numerical techniques, which require the use of the physical model of the material and then they are not easy to generalize $(2,3,4)$.

Our model is based on a wave propagating within a tube where the test specimen is placed at one end. As the surface is not planar, we have split it up into small steps, in such a way that the incidence is normal to the surface, the material is supposed locally reacting, and to estimate the acoustic reflection coefficient, force instead pressure has been used.

\section{THEORY.}

As stated above we will assume the propagation of an acoustical wave in a tube closed in one end by a pulsating piston and at the other end by a sample of absorbent acoustical material. The surface of the test specimen can have any shape, but its rear face has been supposed plane and hard-backed.

When the piston oscillates, the forces generated inside the tube propagate through the fluid mass to reach the sample surface. To the excitation produced by the incident wave front, the material reacts with a force which value is a function of the mecano-acoustical 
characteristics of the material and the surface associated to the force. Due to the shape of the sample's surface the reflected pressure is not constant, varying from one point to another, and consequently acoustic reflection coefficient is not well defined. Thus we define reflection coefficient for such a surface as a relation between forces instead of a relation between pressures, i. e.

$$
R_{T}=\frac{F_{r}}{F_{i}}
$$

being $F_{i}$ the incident force to the surface, and $F_{r}$ the total reflected force exerted by the sample surface to the air. If we approximate the surface by a set of small steps (Figure 1). Then Equation (1) can be particularized for the reflected force by

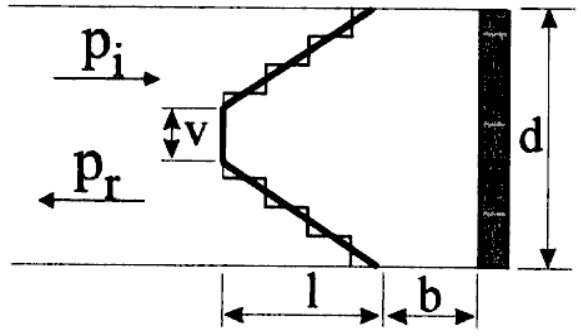

FIGURE 1.

$$
R_{T}=\frac{\sum_{j} p_{r, j} s_{j}}{p_{i} \sum_{j} s_{j}}=\frac{1}{S} \sum_{j} R_{j} s_{j}
$$

where $p_{\mathrm{r}, \mathrm{j}}$ and $s_{\mathrm{j}}$ are the pressure and surface of the $\mathrm{j}$-th element, $R_{j}$ the reflection coefficient associated to an incident plane wave on an elementary surface $s_{j}$ and $p_{i}$ the incident acoustic pressure. Assuming that the material is locally reacting, the reflection coefficient is a function of the acoustic characteristics of the sample, and its thickness. From Equation (2) it can be shown that, if the surface was plane this definition would coincide with the classical one for pressures. In Equation (2), the reflection coefficient $R_{j}$ associated to each step can be calculated through the expression:

$$
R_{j}=\frac{Z_{j}-Z_{0}}{Z_{j}+Z_{0}}
$$

being $Z_{0}$ the air characteristic impedance and $Z_{j}$ the elementary surface impedance of the test material referred to a measuring plane. Its calculus depends on the physical nature of the employed material; for granular ones several constitutive parameters should be determined and also several models have been proposed $(5,6)$.

\section{MEASUREMENTS}

A series of measurements was carried out to test the above expressions within a standing wave tube. The samples were rubber crumb wedges and a cylinder of the same material to be able to test the improvement. Results are shown in Figure 2. It can be observed that wedges smoothes the minima of the acoustic absorption, being the first minimum, the only one that remains almost unaltered. Obviously, the longer the back, the higher the absorption is, for high frequencies. 

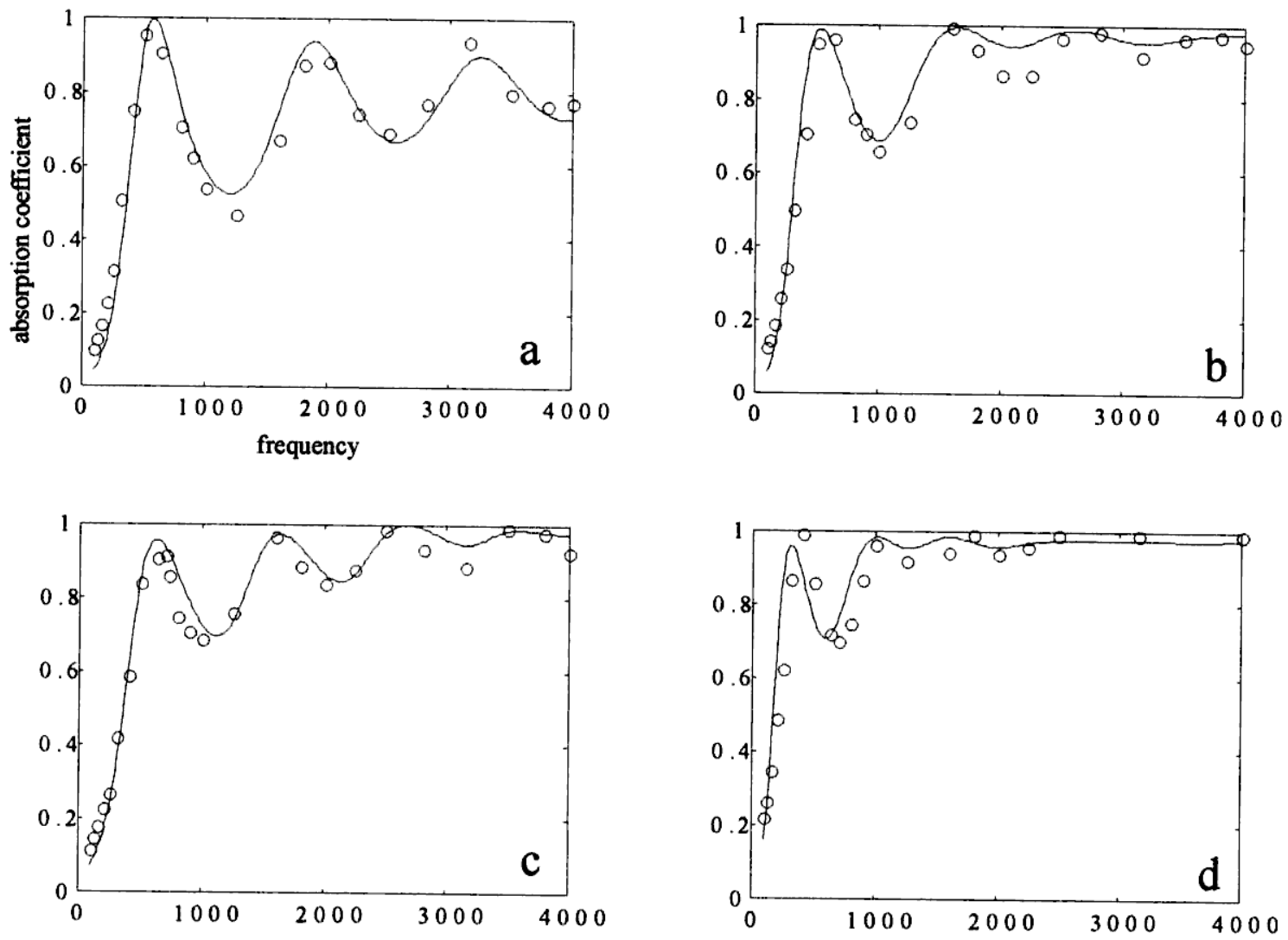

FIGURE 2. Absorption coefficient for different rubber crumb samples . a) cylinder. $d=10 \mathrm{~cm}, b=9 \mathrm{~cm}$. b) wedge. $d=10 \mathrm{~cm}, b=5 \mathrm{~cm}, l=7 \mathrm{~cm}, v=3 \mathrm{~cm}$. c) wedge. $d=10 \mathrm{~cm}, b=2 \mathrm{~cm}, l=11 \mathrm{~cm}, v=3 \mathrm{~cm}$. d) wedge. $d=10 \mathrm{~cm}$, $b=8 \mathrm{~cm}, l=11 \mathrm{~cm}, a=3 \mathrm{~cm}$.

\section{ACKNOWLEDGEMENT}

This work was supported by the Dirección General de Investigación Científica y Técnica (DGICYT), Project Number AMB 98-1029-C04-01

\section{REFERENCES}

1. Beranek, L. L. and Sleeper, H. P. J. Acoust. Soc. Am. 18, 140-150 (1946).

2. Easwaran V. and Munjal M. L. J. Sound and Vib. 160(2), 333-350 (1993)

3. Koidan, W. Hruska, G. R. and Picket, M. A. J. Sound and Vib. 52 1070-1076 (1972)

4. Kang, Y. J. and Bolton, J. S. J. Vib. and Acoust. 118, 498-504 (1996)

5. Pfretzschner, J. and Rodriguez, R. M. Polymer Testing. In press (1999)

6. Champoux, Y. and Stinson, M. R. J. Acoust. Soc. Am. 92 (2), 1120-1131 (1992). 